throughout the event in rave parties, following advice from drug misuse agencies, and the release of antidiuretic hormone by MDMA. Urgent estimation of serum sodium is therefore indicated in this situation.

There is no available antidote for the management of this condition. Supportive treatment starting in the $A \& E$ department and continuing in an intensive care unit is essential. We think that dantrolene should be used early, in addition to cooling measures, if the temperature is greater than $39^{\circ} \mathrm{C}$ or rapidly rising. ${ }^{8}$ As the number of such patients is increasing, we suggest that departments should have an appropriate protocol for cooling these patients, including administration of dantrolene sodium.
1 Cuomo MJ, Dyment PG, Gammino VM. Increasing use of "Ecstasy" (MDMA) and other hallucinogens on a college campus. J Am Coll Health 1994:42:271-4.

2 Henry JA, Jeffreys KJ, Dawling S. Toxicity and deaths from 3,4-methylenedioxymethamphetamine ("ecstasy"). Lancet 1992;340:384-7.

3 O'Connor B. Hazards associated with the recreational drug 'ecstasy'. Br J Hosp Med 1994;52:507-14.

4 Gordon CJ, Watkinson WP, O'Callaghan JP, Miller DB. Effects of 3,4-methylenedioxymethamphetamine on autonomic thermoregulatory responses of the rat. Pharmacol Biochem Behav 1991:38:339-44.

5 Bouchama A. Heat stroke: a new look at an ancient disease. Intensive Care Med 1995;21:623-5.

6 Tehan B, Hardern R, Bodenham A. Hyperthermia associated with 3,4-methylenedioxyethamphetamine ('Eve'). Anaesthesia 1993;48:507-10.

7 Walking on the moon [editorial]. Lancet 1996;347:207.

8 Bodenham AR, Mallick A. New dimensions in toxicology: hyperthermic syndrome following amphetamine derivatives. Intensive Care Med 1996;22:622-4.

\title{
Seat belt injuries and sigmoid colon trauma
}

\author{
E M Eltahir, D Hamilton
}

\begin{abstract}
Colonic seat belt injuries are rare but carry higher mortality rates than small bowel injuries. The case of a 44 year old man is described who had severe sigmoid colon compression injury from his seat belt a few days after a road traffic accident.

(F Accid Emerg Med 1997;14:338-339)
\end{abstract}

Keywords: seat belt; sigmoid compression

\section{Case report}

A 44 year old male driver with no significant past medical history presented to the accident

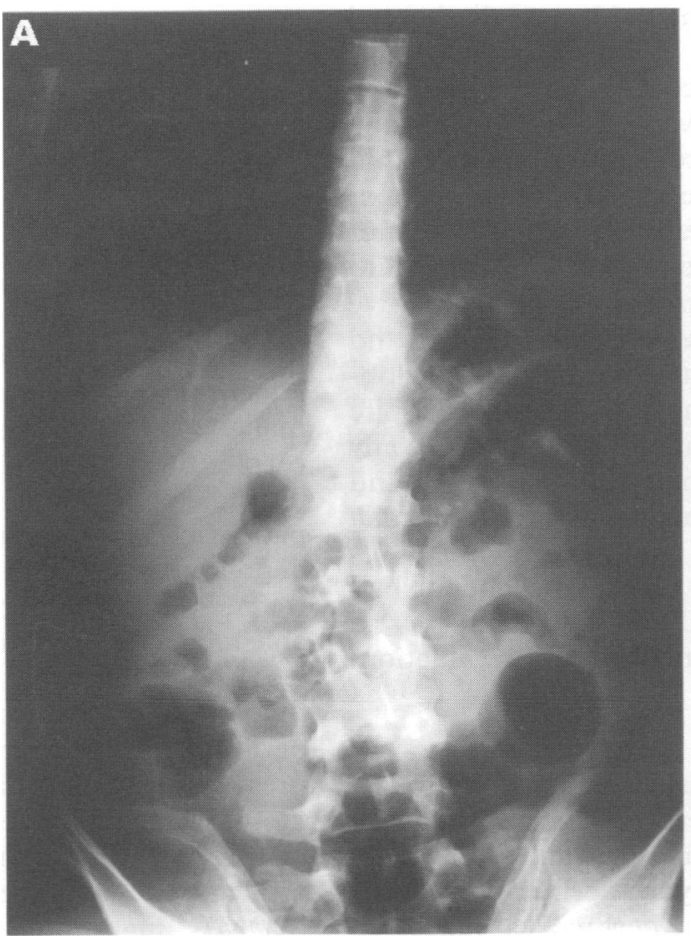

Figure 1 Radiological appearances: $(A)$ erect; (B) supine. and emergency department two days after a road traffic accident with increasing abdominal pain and constipation.

He was febrile, dehydrated, and abdominal examination showed severe peritonitis. There was a faint resolving bruise over the lateral margin of the left iliac fossa. Digital rectal examination was normal.

He was the driver in a road traffic accident when he skidded and hit a lamp post. The front passenger died of severe head injury and two of the three back seat passengers sustained variable physical injuries. He was treated in another city hospital for minor facial wounds

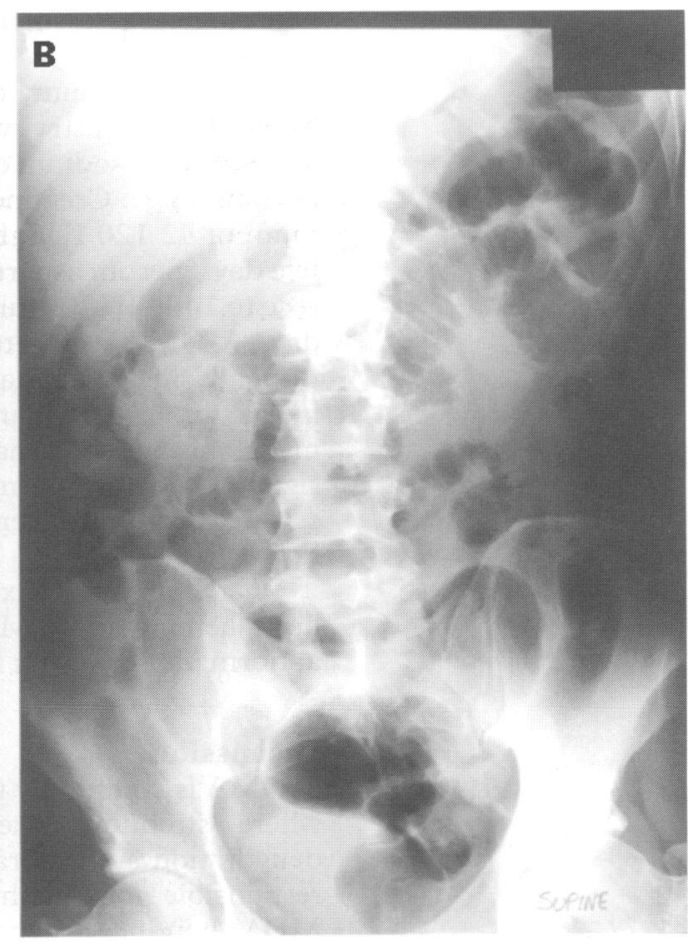

\author{
Accepted for publication \\ 28 May 1997 \\ Department of \\ General Surgery, \\ Royal Alexandra \\ 9PN, UK \\ E M Eltahir \\ D Hamilton \\ Correspondence to: \\ Mr E Eltahir.
}


but took his own discharge 12 hours after admission. At that time an abdominal ultrasound was negative and full blood count was normal.

On the present admission routine blood examination revealed a leucocytosis of $23.1 \times 10^{9} / 1$, haemoglobin of $15.0 \mathrm{~g} / 1$, and normal serum amylase and liver function tests. Plain abdominal radiographs showed localised dilated loops of bowl in the upper quadrant to the left with a few fluid levels. There was no free intraperitoneal gas and chest $x$ ray showed no rib fractures.

After three hours of resuscitation, laparotomy was undertaken through a midline incision. There was a three inch long ischaemic segment in the upper sigmoid colon, which was severely bruised with areas of gangrene and necrosis. Its surface was thin in places and about to perforate. Nine inches of colon were resected. Five inches proximal to this, the lower descending colon showed a five inch long segment which was contused, indurated, and thickened but viable. Both areas of damage corresponded with the position of seat belt straps. A Hartmann's procedure was carried out without difficulty. The rest of the laparotomy was normal. Postoperative recovery was uneventful.

Histopathology of the resected segment showed extensive mucosal necrosis with focal ulceration and transmural acute inflammation, with submucosal oedema and vascular congestion. Parts of the wall were completely gangrenous and the appearance was in keeping with ischaemic injury.

\section{Discussion}

The use of seat belts has reduced the incidence and severity of road traffic accidents injuries. ${ }^{1}$ Nevertheless, seat belt themselves can cause serious injuries. In abdominal trauma the underlying viscera may be crushed between the seat belt, the abdominal wall, and the lumbar vertebrae if the anterior abdominal muscles are relaxed at the moment of impact, or if the viscera are distended or pathologically enlarged. Late presentation occurs if the bowel is not ruptured immediately. In such cases sepsis may develop, with increased mortality. A high index of suspicion is necessary to avoid diagnostic delays. Serious injuries to other passengers are evidence of the violence of the accident, as is abdominal wall bruising.

Patients at risk should be admitted to hospital and regular observations and serial examinations performed. A variety of further investigations including minilaparoscopy may be helpful.

1 Howdieshell TR, Delaurier G. An unusual injury of the sigmoid colon produced by seat belt trauma. Am Sur 1993;59:355-8.

\title{
Saturday night blue - a case of near fatal poisoning from the abuse of amyl nitrite
}

\author{
Tom Stambach, Kevin Haire, Neil Soni, Jeremy Booth
}

\section{Chelsea and}

Westminster Hospital, London SW10:

Department of

Anaesthetics

T Stambach

K Haire

Intensive Care Unit

N Soni

Accident and

Emergency

Department

$\mathrm{S} J$ Booth

Correspondence to:

Mr S Jeremy Booth, Accident and Emergency Department,

Chelsea and Westminster

Hospital, Fulham Road,

London SW10 9NH.

Accepted for publication 11 March 1997

\begin{abstract}
A case of severe methaemoglobinaemia caused by the abuse of volatile nitrites is reported. The agents are commonly abused, but this complication is rare. The clinical presentation can make diagnosis difficult; however, the subsequent treatment needs to be rapid to avoid serious morbidity or mortality. This report presents the clinical picture and the background information leading to the detection and treatment of this unusual problem.

(F Accid Emerg Med 1997;14:339-340)
\end{abstract}

Keywords: methaemoglobinaemia; volatile nitrites; diagnosis; treatment.

The use of volatile nitrites as drugs of abuse is commonplace. We present here an uncommon but potentially fatal complication of their use. Rapid diagnosis and treatment in this case resulted in a full recovery and we have highlighted the important features of this uncommon toxicological problem.

\section{Case report}

An unknown white woman in her early twenties was found collapsed outside a police station. She was initially thought to be dead, as she was blue, there was no obvious breathing, and no pulse was palpable. An ambulance was called and on their arrival the crew detected a pulse, some respiration was evident, and she was treated with $100 \%$ oxygen and a total of $1.6 \mathrm{mg}$ naloxone. She remained blue, her respiration was unchanged, and a pulse oximeter read $83 \%$.

On arrival in accident and emergency (A\&E) she was profoundly cyanosed with rapid irregular respiration, pulse $130 / \mathrm{min}$, blood pressure $80 / 30 \mathrm{~mm} \mathrm{Hg}$, and marked peripheral vasoconstriction. Her pupils were dilated but weakly reactive, Glasgow coma score 5 . She was actively resuscitated with a rapid infusion of colloid and was intubated and ventilated with $100 \%$ oxygen. Arterial blood gases 